\title{
Femoral Nerve Block versus Adductor Canal Block for Analgesia after Total Knee Arthroplasty
}

\author{
In Jun Koh, MD ${ }^{1,2}$, Young Jun Choi, $\mathrm{MD}^{1,2}$, Man Soo Kim, $\mathrm{MD}^{1,2}$, Hyun Jung Koh, $\mathrm{MD}^{3}$, Min Sung Kang, $\mathrm{MD}^{1}$, \\ and Yong In, $\mathrm{MD}^{1,2}$ \\ ${ }^{1}$ Department of Orthopaedic Surgery, Seoul St. Mary's Hospital, Seoul; ${ }^{2}$ Department of Orthopaedic Surgery, College of Medicine, The Catholic University of Korea, \\ Seoul; ${ }^{3}$ Department of Anesthesia and Pain Medicine, Seoul St. Mary's Hospital, Seoul, Korea
}

Inadequate pain management after total knee arthroplasty (TKA) impedes recovery, increases the risk of postoperative complications, and results in patient dissatisfaction. Although the preemptive use of multimodal measures is currently considered the principle of pain management after TKA, no gold standard pain management protocol has been established. Peripheral nerve blocks have been used as part of a contemporary multimodal approach to pain control after TKA. Femoral nerve block (FNB) has excellent postoperative analgesia and is now a commonly used analgesic modality for TKA pain control. However, FNB leads to quadriceps muscle weakness, which impairs early mobilization and increases the risk of postoperative falls. In this context, emerging evidence suggests that adductor canal block (ACB) facilitates postoperative rehabilitation compared with FNB because it primarily provides a sensory nerve block with sparing of quadriceps strength. However, whether ACB is more appropriate for contemporary pain management after TKA remains controversial. The objective of this study was to review and summarize recent studies regarding practical issues for $\mathrm{ACB}$ and comparisons of analgesic efficacy and functional recovery between ACB and FNB in patients who have undergone TKA.

Keywords: Knee, Arthroplasty, Pain management, Nerve block, Saphenous nerve, Femoral nerve

\section{Introduction}

Total knee arthroplasty (TKA) involves extensive bone resection and soft tissue manipulation, and patients can experience severe pain during the early postoperative period ${ }^{1-5)}$. Appropriate pain management after TKA allows for faster recovery, reduces the risk of postoperative complications, and improves patient satisfaction. Although the preemptive use of multimodal measures is currently accepted as a principle of pain management after

Received July 7, 2016; Revised August 13, 2016;

Accepted October 3, 2016

Correspondence to: Yong In, MD

Department of Orthopaedic Surgery, Seoul St. Mary's Hospital, College of Medicine, The Catholic University of Korea, 222 Banpo-daero, Seocho-gu, Seoul 06591, Korea

Tel: +82-2-2258-6111, Fax: +82-2-535-9834

E-mail: iy1000@catholic.ac.kr

This is an Open Access article distributed under the terms of the Creative Commons Attribution Non-Commercial License (http://creativecommons.org/licenses/by-nc/4.0/) which permits unrestricted non-commercial use, distribution, and reproduction in any medium, provided the original work is properly cited.
TKA, no gold standard pain management protocol has been established. Contemporary pain management regimens following TKA include oral analgesics, periarticular injection, peripheral nerve blocks (PNBs), and intravenous patient-controlled analgesia $^{4-7)}$. As PNBs provide effective and synergistic pain relief when used as part of a multimodal regimen, they are considered to be an essential part of the current multimodal pain management protocol following $\mathrm{TKA}^{1,7-9)}$.

Given excellent pain relief and the opioid sparing effect, femoral nerve block (FNB) is commonly used as an analgesic modality and is considered the standard PNB in patients undergoing TKA $^{10}$. However, FNB is followed by a significant decrease in quadriceps muscle strength, resulting in delayed mobilization, which is associated with the potential risk of falling ${ }^{11-16)}$. Recently, as the length of stay (LOS) in hospital has been shortened by the performance of TKA on an outpatient basis, a potent analgesia that preserves motor strength during early rehabilitation is becoming increasingly accepted as an essential part of the current perioperative protocol following TKA. In this context, a growing body of evidence supports the use of an adductor canal block 
(ACB) that offers almost pure sensory block with minimal motor involvement as part of a multimodal approach to pain control after TKA ${ }^{17-32)}$. However, a limited number of studies have examined the anatomy and infiltration technique of $\mathrm{ACB}$. In addition, studies comparing ACB to FNB in terms of analgesic efficacy and functional recovery in patients undergoing TKA remain limited.

Therefore, this comprehensive review was performed to 1) review current studies regarding the anatomy, infiltration technique, analgesic efficacy, and functional recovery of ACB for pain management following TKA and 2) compare the analgesic efficacy and functional recovery of ACB and FNB in patients who have undergone TKA.

\section{Methods}

This comprehensive review included all types of study design, including randomized controlled trials (RCTs), retrospective comparative clinical trials, systematic reviews, and meta-analyses investigating the anatomy, infiltration technique, analgesic efficacy, and functional recovery of ACB, as well as comparison of analgesic efficacy and functional recovery between ACB and FNB in patients undergoing TKA. English language studies were identified by searching PubMed, MEDLINE, and EMBASE, and subsequently by searching the bibliographies of all relevant retrieved articles. The search included publications that 1) were publicly assessable on the internet; 2) were published in English after 2006; 3) presented the anatomy of the adductor canal; 4) presented the ACB infusion technique; 5) reported analgesic efficacy or functional recovery of $\mathrm{ACB}$; and 6) reported comparative data regarding analgesic efficacy or functional recovery between $\mathrm{ACB}$ and FNB, as well as one of the followings: pain level, opioid consumption, frequency of opioid-related side effects, satisfaction, quadriceps strength, mobilization ability, risk of falls, and LOS. The following terms were used for the initial literature search: "adductor canal" OR "adductor canal block" OR "femoral nerve block" OR "knee arthroplasty" OR "knee replacement" OR "total knee arthroplasty" OR "total knee replacement" OR "TKA" OR "TKR". Two authors (CYJ and KMS) of this study reviewed the full texts of all identified articles, and studies that did not report on any of the outcome variables listed above were excluded. The authors discussed any difference of opinion on study inclusion until consensus was achieved. Of the 153 identified articles, 56 duplicates were removed and 62 were excluded because they did not meet the inclusion criteria. Thus, the detailed full articles of 35 studies were reviewed. Of these, 3 were excluded because the study population did not receive TKA. Finally, 32 articles remained in this systematic review (Fig. 1). This study was exempted from Institutional Review Board review because it did not involve human subjects.

\section{Results}

\section{Practical Issues for ACB}

\section{1) Anatomy}

The adductor canal, also known as the subsartorial or Hunter canal, is an aponeurotic tunnel that begins at the apex of the femoral triangle and ends at the adductor hiatus, serving as a passageway for the major neurovascular bundle from the femoral triangle to the popliteal fossa (Fig. 2). It is roofed by the vasto-adductor membrane, also known as the anteromedial intermuscular septum or the subsartorial fascia, which is a strong aponeurosis between the adductor muscles and the vastus medialis muscle. It contains the femoral vessels, the saphenous nerve (SN), and the nerve to the vastus medialis muscle (NVM) ${ }^{33-37)}$. One recent cadaveric study showed that the SN and NVM were consistently present, whereas branches of the anterior obturator nerve were inconsistently present. In addition, the NVM contributed significantly to innervation of the knee capsule through intramuscular,

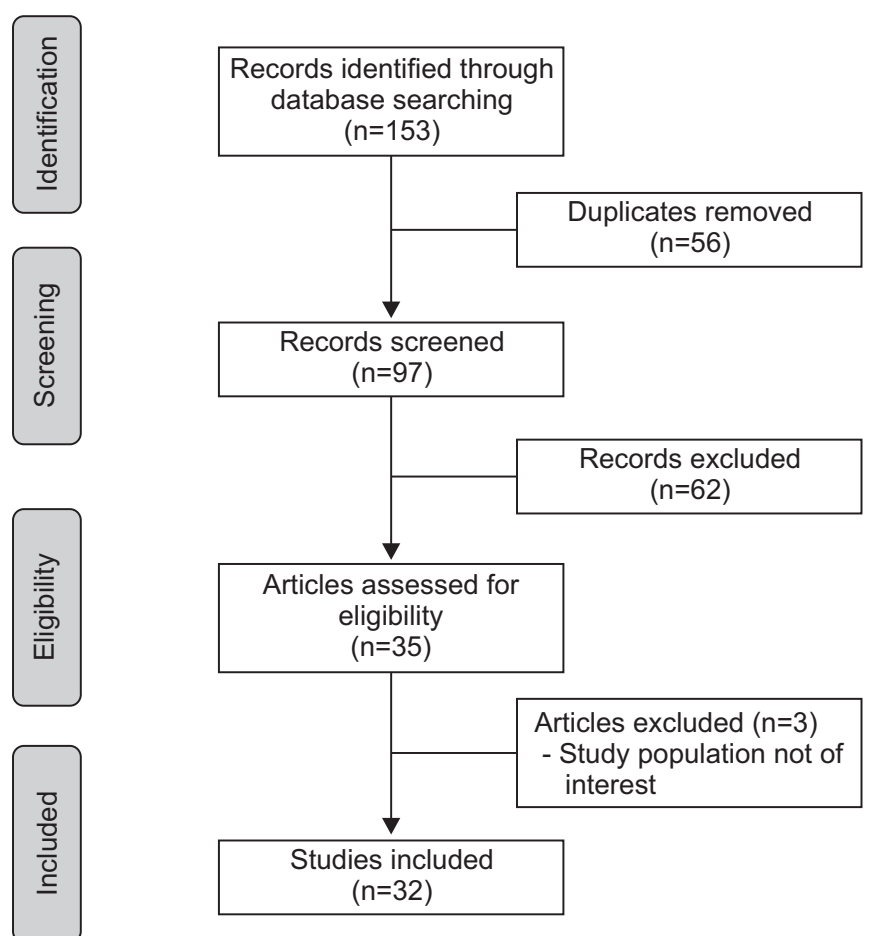

Fig. 1. Flowchart of the search strategy. 


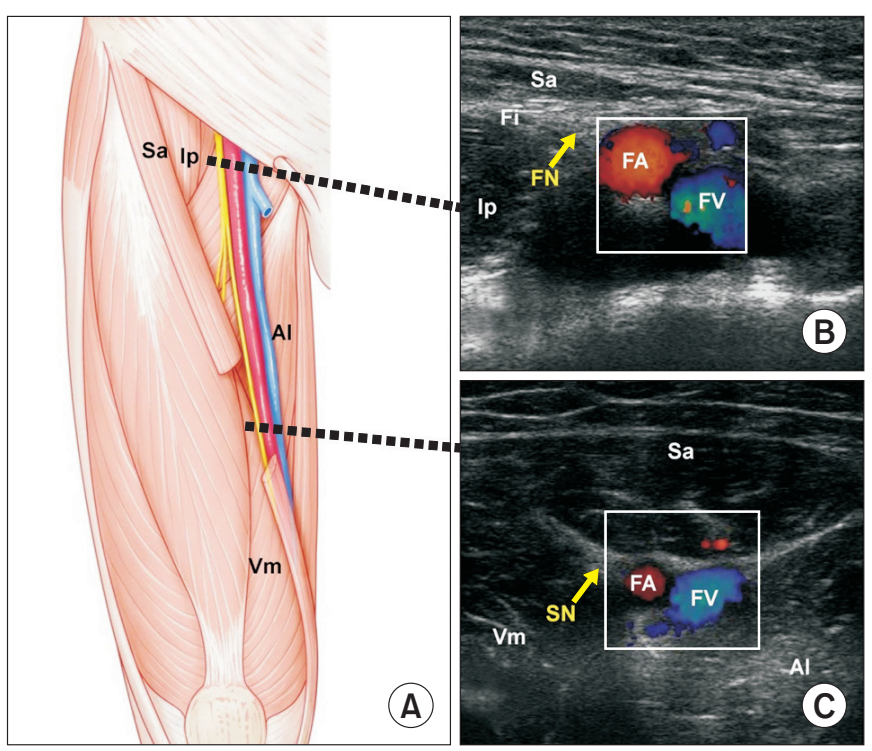

Fig. 2. (A) Schematic drawing of the anterior aspect of right thigh. The mid portion of the sartorius muscle was cut to show the inside of the adductor canal. (B) Cross-sectional ultrasonography image at the apex of the femoral triangle. (C) Cross-sectional ultrasonography image of the adductor canal. FN: femoral nerve, FA: femoral artery, FV: femoral vein, $\mathrm{SN}$ : saphenous nerve, Sa: sartorius muscle, Ip: iliopsoas muscle, Fi: fascia iliaca, $\mathrm{Vm}$ : vatus medialis muscle, $\mathrm{Al}$ : adductor longus muscle.

extramuscular, and deep genicular nerves ${ }^{33)}$.

\section{2) Technique}

Recent advances in high quality, portable ultrasound technology have made ultrasound-guided ACB a standard practice in most institutions. A high frequency linear ultrasound transducer was placed transverse to the longitudinal axis of the extremity at the mid-thigh, approximately midway along the distance between the iliac spine and the patella. Next, the femoral artery was found underneath the sartorius muscle with the vein in the short axis. At this level, the SN, which was shown as a hyperechoic structure, was placed lateral to the artery in the adductor canal (Fig. 2). For single shot adductor canal block (SACB), local anesthetics such as $15 \mathrm{~mL}$ of $0.25 \%$ to $0.5 \%$ bupivacaine or $15 \mathrm{~mL}$ to $30 \mathrm{~mL}$ of $0.2 \%$ to $0.5 \%$ ropivacaine was infiltrated around the $\mathrm{SN}^{24-27,31,34,38-44)}$. For continuous adductor canal block (CACB), a 17- or 18-Tuohy cannula was inserted from the lateral side of the transducer, through the sartorius muscle, with the tip placed lateral to the artery. Normal saline or local anesthetics were then injected to expand the canal, and a flexible catheter was inserted through the cannula. Finally, an additional dosage of local anesthetics was injected as per the protocol ${ }^{19,21-23,28-30,32,35,45)}$.

\section{3) Analgesic efficacy}

After performing ACB, complete sensory loss of the medial, anterior, and lateral aspects of the knee extending from the superior pole of the patella to the proximal tibia, with no noticeable quadriceps strength loss, has been reported ${ }^{20)}$. In addition, previous RCTs showed that ACB significantly decreased postoperative pain and opioid consumption during the first 24 hours in patients who underwent arthroscopic surgery ${ }^{46-48)}$. Moreover, recent RCTs showed that CACB provided excellent analgesia and opioid sparing effects during the first 48 hours in patients who underwent TKA compared with placebo ${ }^{19,34,35)}$. On the other hand, CACB with catheter use has been reported to offer superior analgesic effects, but similar functional recovery, compared with $\mathrm{SACB}^{45)}$.

\section{4) Functional recovery}

Jaeger et al. ${ }^{49)}$ reported that ACB preserved quadriceps strength and improved ambulation compared with FNB. In that study, quadriceps strength decreased $8 \%$ from baseline following ACB, but $49 \%$ following $\mathrm{FNB}$, in 11 randomized healthy volunteers. Another healthy volunteer trial also showed that quadriceps strength and balance scores were similar to baseline following $\mathrm{ACB}$, but decreased significantly following $\mathrm{FNB}^{50}$. In addition, patients receiving CACB showed superior quadriceps strength and distance ambulated compared with those receiving placebo following $\mathrm{TKA}^{19)}$. CACB also resulted in significantly enhanced ambulation ability, as assessed with the Timed Up and Go (TUG) test $^{34)}$.

\section{Comparison of Clinical Results between ACB and FNB}

In this study, we reviewed 12 clinical trials (Table 1) and 6 metaanalyses (Table 2). Seven of 12 clinical trials examined CACB with catheter use, and five studies examined SACB.

\section{1) Clinical trials}

\section{(1) Analgesic efficacy}

\section{Continuous block with catheter}

Analgesic efficacy within 2 postoperative days (PODs) was comparable between $\mathrm{ACB}$ and FNB in terms of pain level, opioid consumption, and frequency of opioid-related side effects across five $\mathrm{RCTs}^{21,22,28,29,32)}$ and two retrospective clinical trials ${ }^{23,30)}$, excluding one RCT that documented better pain relief in the FNB group on POD $0^{21}$. In addition, one RCT reported similar satisfaction between groups ${ }^{21}$. On the other hand, ACB was reported to offer provider benefits by decreasing catheter-related provider 


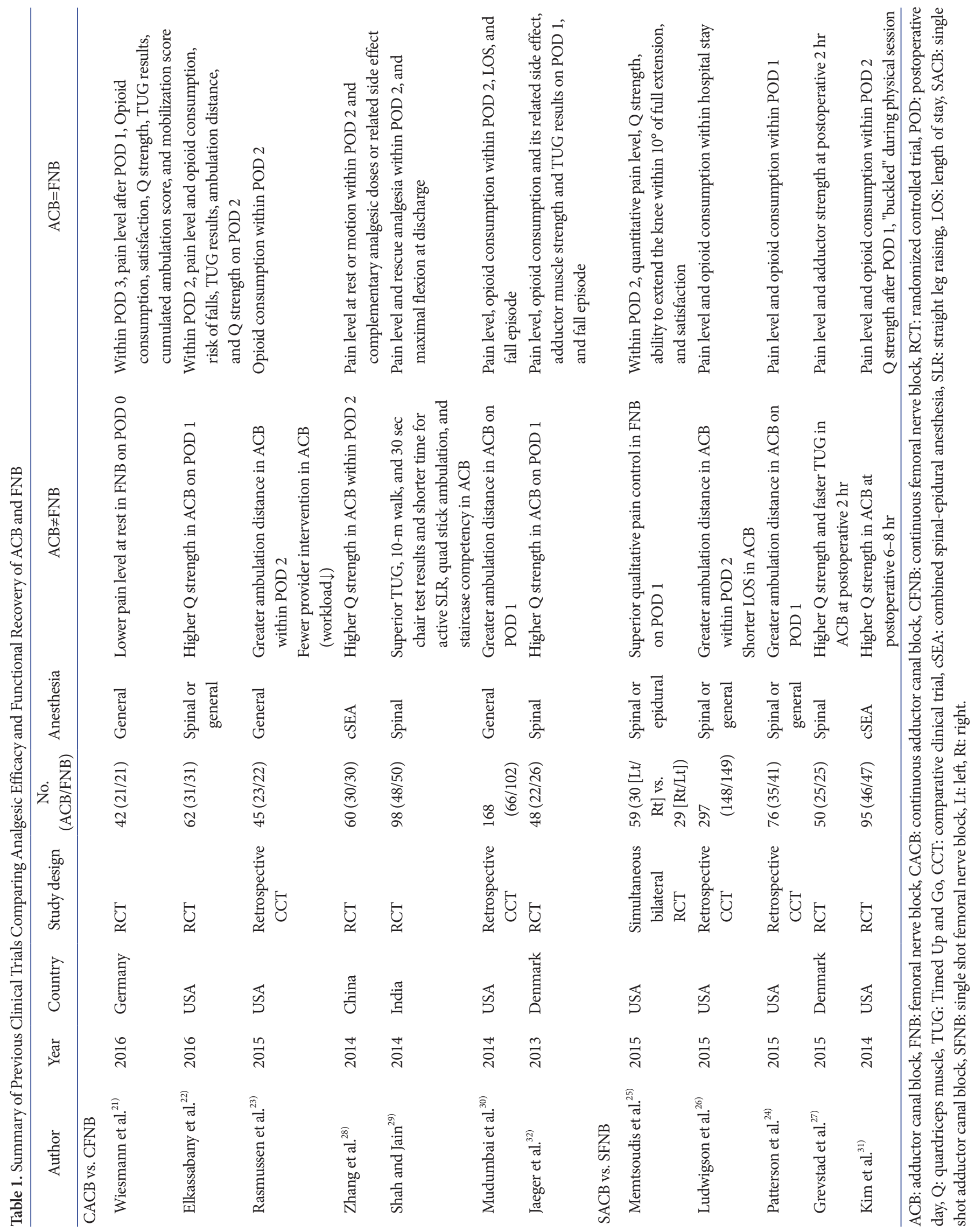


Knee Surg Relat Res, Vol. 29, No. 2, Jun. 201791

Table 2. Summary of Previous Meta-Analyses Comparing Analgesic Efficacy and Functional Recovery of ACB and FNB

\begin{tabular}{|c|c|c|c|c|c|c|}
\hline Author & Year & Study (no.) & $\mathrm{ACB} / \mathrm{FNB}$ & $\begin{array}{l}\text { Continuous/ } \\
\text { single shot }\end{array}$ & $\mathrm{ACB}>\mathrm{FNB}$ & $\mathrm{ACB}=\mathrm{FNB}$ \\
\hline Zhao et al. ${ }^{44)}$ & 2016 & $\begin{array}{l}5 \mathrm{RCTs}^{25,27,29,31,32)} \\
(348)\end{array}$ & $200 / 207$ & $146 / 204$ & Q strength and mobilization ability & $\begin{array}{l}\text { Pain level, opioid consumption } \\
\text { and related SE, satisfaction, and } \\
\text { adductor strength }\end{array}$ \\
\hline Li et al. ${ }^{43)}$ & 2016 & $\begin{array}{l}8 \mathrm{RCTs}^{25,27-29,31,32,49,50)} \\
\quad(434)\end{array}$ & $249 / 255$ & $222 / 212$ & $\begin{array}{l}\text { Resting pain within postoperative } \\
8-24 \mathrm{hr} \\
\text { Q strength and mobilization ability }\end{array}$ & $\begin{array}{l}\text { Pain level after POD 2, opioid } \\
\text { consumption, satisfaction, } \\
\text { adductor strength, and tourniquet } \\
\text { time }\end{array}$ \\
\hline Kuang et al. ${ }^{41)}$ & 2015 & $\begin{array}{l}4 \operatorname{RCTs}^{28,29,31,32)} \\
3 \operatorname{CCTs}^{24,26,30)}(828)\end{array}$ & $383 / 445$ & $374 / 468$ & $\begin{array}{l}\text { Resting pain within postoperative } \\
24 \mathrm{hr} \text {, postoperative nausea and } \\
\text { vomiting, mobilization ability and } \\
\text { ambulation distance, and LOS }\end{array}$ & $\begin{array}{l}\text { Pain level and opioid consumption } \\
\text { after POD } 2\end{array}$ \\
\hline Hussain et al. ${ }^{40)}$ & 2016 & $\begin{array}{l}6 \operatorname{RCTs}^{25,27-29,31,32)} \\
(408)\end{array}$ & $230 / 237$ & $206 / 204$ & $\begin{array}{l}\text { Q strength within postoperative } \\
8-24 \mathrm{hr}\end{array}$ & $\begin{array}{l}\text { Pain level at rest or motion within } \\
\text { POD 2, and complement analgesic } \\
\text { doses or related SE }\end{array}$ \\
\hline Dong et al. ${ }^{39)}$ & 2016 & $\begin{array}{l}6 \mathrm{RCTs}^{25,27-29,31,32)} \\
2 \mathrm{CCTs}^{24,30)}(751)\end{array}$ & $360 / 391$ & $374 / 280$ & - & $\begin{array}{l}\text { Pain level, opioid consumption } \\
\text { and related SE, Q and adductor } \\
\text { strength, and LOS }\end{array}$ \\
\hline $\mathrm{Li}$ and $\mathrm{Ma}^{42)}$ & 2016 & $\begin{array}{l}7 \mathrm{RCTs}^{25,27-29,31,32)} \\
2 \mathrm{CCTs}^{24,30)}(639)\end{array}$ & $295 / 344$ & $374 / 318$ & Q strength on POD 1-2 & $\begin{array}{l}\text { Pain level, opioid consumption and } \\
\text { related SE, risk of falls, and LOS }\end{array}$ \\
\hline
\end{tabular}

ACB: adductor canal block, FNB: femoral nerve block, RCT: randomized controlled trial, Q: quardriceps muscle, SE: side effect, POD: postoperative day, CCT: comparative clinical trial, LOS: length of stay.

interventions per patient compared with $\mathrm{FNB}^{23)}$.

\section{Single shot block}

Pain level and opioid consumption did not differ between groups in three RCTs ${ }^{25,27,31)}$ and two retrospective clinical tri$\operatorname{als}^{24,26)}$. One simultaneous randomized bilateral trial showed comparable quantitative pain level within 2 PODs, but superior pain relief in the FNB group on POD $1^{25)}$. In addition, no between-group difference in satisfaction was reported ${ }^{25)}$.

\section{(2) Functional recovery}

\section{Continuous block with catheter}

Most studies documented greater quadriceps strength and ambulation distance in ACB groups on POD 1 or $2^{22,23,28-30,32)}$; however, the superiority of ACB was limited to POD $2^{21,22)}$. Authors of three RCTs reported comparable TUG test outcomes between groups ${ }^{21,22,32)}$, whereas one study documented superior results in an $\mathrm{ACB}$ group ${ }^{29)}$. Meanwhile, one trial showed a statistical trend toward greater fall risk in the FNB group ( $\mathrm{p}=0.06)$, but the sample of that study was limited to 31 patients per group ${ }^{22}$. Another study showed no difference in the frequency of fall episodes between group ${ }^{30}$. Finally, studies produced contradictory results regarding other ambulation ability assessment and functional recovery variables, such as the $10-\mathrm{m}$ walk test, 30 -second chair test, time for quad stick ambulation or staircase competency ${ }^{29)}$, cumulated ambulation score, and mobilization score ${ }^{211}$.

\section{Single shot block}

Greater quadriceps strength in ACB groups at postoperative 2 hours $^{27)}, 6-8$ hours $^{31)}$, and longer ambulation distance on POD $1^{24)}$ or $2^{26)}$ were reported. However, quadriceps strength on POD $1^{31)}$ or $2^{25)}$ did not differ. One RCT documented faster TUG performance in the ACB group at postoperative 2 hours, and a retrospective study showed a shorter reduced LOS in hospital in the ACB group ${ }^{26)}$.

\section{2) Meta-analyses}

\section{(1) Analgesic efficacy}

Comparable pain level, opioid consumption, and opioid-related side effects were reported in all six meta-analyses reviewed in this study ${ }^{39-44)}$, excluding two studies reporting superior resting pain in ACB groups on POD $1^{41,43)}$. In addition, similar satisfaction was reported in two studies ${ }^{43,44)}$. 


\section{(2) Functional recovery}

Four of the six meta-analyses showed greater quadriceps muscle strength in ACB recipients on POD $1^{40,42-44)}$, but one study found no between-group difference ${ }^{39}$. In addition, similar adductor muscle strength was reported in three studies ${ }^{39,43,44)}$. Two studies showed similar LOSs ${ }^{39,42)}$, whereas one study found a shorter LOS in the ACB group ${ }^{41}$. Similar risks of falls were also reported ${ }^{42}$.

\section{Discussion}

As the emphasis has been on faster recovery during the early postoperative period, recent trends in pain management protocols following TKA have shifted toward effective analgesia with limited motor involvement. Given the excellent analgesic effect, FNB is a commonly used modality as part of TKA pain control regimens and is considered to be the gold standard for postoperative analgesia after TKA. However, it may reduce quadriceps strength, which is essential for early mobilization and is associated with an increased risk of postoperative falls. Thus, ACB has emerged as a reasonable alternative to FNB that produces a predominantly sensory block with greater quadriceps strength preservation. However, whether ACB can be used as part of TKA multimodal pain management in clinical practice remains unclear, and comparisons of analgesic efficacy and functional recovery between $\mathrm{ACB}$ and FNB remain limited. This comprehensive review was conducted 1) to explore practical issues for $\mathrm{ACB}$ and 2) to compare analgesic efficacy and functional recovery between $\mathrm{ACB}$ and FNB in patients undergoing TKA.

The findings of this study need to be interpreted with considerations of the following limitations. First, as we only performed an extensive search, we could not identify statistical significance or concrete consensus. In addition, heterogeneities among studies regarding drug composition, infiltration techniques, concomitant pain management protocols, and outcome variables make it difficult to judge the practical value of ACB and we could not find any clinical relevance among infiltration techniques. Future studies that investigate these issues in more identical manners are required. Second, we did not compare the cost effectiveness, time required, or anesthesiologist's learning curve for satisfactory blockade. These data would increase our understanding of the appropriateness of ACB following TKA. Future studies investigating these issues in more detail are required. Third, we did not evaluate data on national health insurance systems, which may regulate postoperative pain management modalities after TKA. This difference in national health systems among countries should be considered before estimation of adjunct analgesic mo- dality after TKA. Despite these limitations, we believe that this study provides valuable information on the usefulness of $\mathrm{ACB}$ in pain management after TKA.

Our findings in this review indicate that $\mathrm{ACB}$ is one of the most useful analgesic modalities in contemporary perioperative management protocols that focus on rapid recovery after knee surgery. ACB can be performed easily with recently introduced high quality, portable ultrasound technology during surgeries around the knee joint, with high success rates ${ }^{27,29,31)}$. In addition, it provided excellent pain relief around the knee joint compared with placebo ${ }^{19,20,35)}$ and preserved motor strength with minimal differences from baseline ${ }^{18,49,50}$. Indeed, multiple recent studies showed that ACB offered satisfactory analgesic effects with wellpreserved mobilization ability in patients who had undergone arthroscopic surgery or TKA ${ }^{19,33,35,45-48)}$. These findings, together with current trends of perioperative protocols toward rapid recovery after TKA, suggest that ACB should be taken into account as part of a contemporary multimodal approach to pain control after TKA. However, more detailed neural components inside the adductor canal and analgesic effects of each neural component following $\mathrm{ACB}$ should be defined, and the optimal type and amount of local anesthetics and mode of infusion should be determined, to enable wide use of $\mathrm{ACB}$ as an adjunctive analgesic modality in pain management after TKA.

$\mathrm{ACB}$, which offers almost pure sensory blockade, seems to be a reasonable alternative to FNB that leads to substantial reduction in quadriceps muscle strength, as part of a current TKA pain control protocol. All studies included in final analyses in this review showed comparable analgesic efficacy in terms of pain level and opioid consumption between $\mathrm{ACB}$ and $\mathrm{FNB}^{21-32,39-44)}$. In addition, most studies documented superior quadriceps strength and mobilization ability during the first 24 hours after TKA for ACB compared with FNB ${ }^{22,27,28,30-32,40-44)}$. Moreover, ACB catheters required fewer provider interventions per patient, thus decreasing the workload compared with FNB catheters ${ }^{23)}$. The findings of this study suggest that ACB is a more appropriate analgesic modality than FNB in patients undergoing current multimodal perioperative protocols after TKA. However, superior functional recovery in ACB groups was limited to the 24-48-hour period after TKA. In addition, although ACB offered comparable pain relief with preserved motor strength, patient satisfaction did not differ ${ }^{21,25,43,44)}$. Moreover, there was no evidence that ACB reduced the risk for postoperative falls, which may be a fatal complication of $\mathrm{FNB}^{22,30-32,42)}$, or $\operatorname{LOS}^{30,39,42)}$ compared with FNB. Heterogeneity among studies in reporting outcome variables made uniform comparison difficult, and further studies are needed to determine 
whether ACB provides superior functional recovery compared with FNB. In addition, the clinical relevance of motor strength during the immediate postoperative period (within 24 hours after TKA) should be determined. Further studies with realistic sample sizes and consistent outcome variables are required to determine whether ACB provides clinically relevant functional recovery compared with FNB.

\section{Conclusions}

Current evidence supports that ACB provides comparable analgesic efficacy and facilitates earlier mobilization by sparing quadriceps strength compared with FNB. Based on current trends in perioperative protocols toward early rehabilitation following TKA, ACB may be a reasonable alternative to FNB as part of a contemporary multimodal pain management protocol after TKA. However, more detailed definition of the neural anatomy inside the adductor canal and determination of the optimal ACB infiltration technique are needed. In addition, further studies with realistic sample sizes are required to determine whether $\mathrm{ACB}$ could provide clinically relevant functional recovery in patients undergoing TKA compared with FNB.

\section{Conflict of Interest}

No potential conflict of interest relevant to this article was reported.

\section{Acknowledgments}

We thank Han Suk Cho, MD for serving as our model during ultrasonography.

\section{References}

1. Korean Knee Society. Guidelines for the management of postoperative pain after total knee arthroplasty. Knee Surg Relat Res. 2012;24:201-7.

2. Sinatra RS, Torres J, Bustos AM. Pain management after major orthopaedic surgery: current strategies and new concepts. J Am Acad Orthop Surg. 2002;10:117-29.

3. Koh IJ, Chang CB, Lee JH, Jeon YT, Kim TK. Preemptive low-dose dexamethasone reduces postoperative emesis and pain after TKA: a randomized controlled study. Clin Orthop Relat Res. 2013;471:3010-20.

4. Koh IJ, Kang YG, Chang CB, Do SH, Seong SC, Kim TK.
Does periarticular injection have additional pain relieving effects during contemporary multimodal pain control protocols for TKA?: a randomised, controlled study. Knee. 2012; 19:253-9.

5. Koh IJ, Kang YG, Chang CB, Kwon SK, Seo ES, Seong SC, Kim TK. Additional pain relieving effect of intraoperative periarticular injections after simultaneous bilateral TKA: a randomized, controlled study. Knee Surg Sports Traumatol Arthrosc. 2010;18:916-22.

6. Koh IJ, Kang YG, Chang CB, Song J, Jeon YT, Kim TK. Use of reduced-dose periarticular injection for pain management in simultaneous bilateral total knee arthroplasty. J Arthroplasty. 2012;27:1731-6.

7. Grosu I, Lavand'homme P, Thienpont E. Pain after knee arthroplasty: an unresolved issue. Knee Surg Sports Traumatol Arthrosc. 2014;22:1744-58.

8. Pagnotto MR, Pagnano MW. Multimodal pain management with peripheral nerve blocks for total knee arthroplasty. Instr Course Lect. 2012;61:389-95.

9. Parvizi J, Miller AG, Gandhi K. Multimodal pain management after total joint arthroplasty. J Bone Joint Surg Am. 2011;93:1075-84.

10. Paul JE, Arya A, Hurlburt L, Cheng J, Thabane L, Tidy A, Murthy Y. Femoral nerve block improves analgesia outcomes after total knee arthroplasty: a meta-analysis of randomized controlled trials. Anesthesiology. 2010;113:114462.

11. Pelt CE, Anderson AW, Anderson MB, Van Dine C, Peters CL. Postoperative falls after total knee arthroplasty in patients with a femoral nerve catheter: can we reduce the incidence? J Arthroplasty. 2014;29:1154-7.

12. Memtsoudis SG, Dy CJ, Ma Y, Chiu YL, Della Valle AG, Mazumdar M. In-hospital patient falls after total joint arthroplasty: incidence, demographics, and risk factors in the United States. J Arthroplasty. 2012;27:823-8.

13. Chan MH, Chen WH, Tung YW, Liu K, Tan PH, Chia YY. Single-injection femoral nerve block lacks preemptive effect on postoperative pain and morphine consumption in total knee arthroplasty. Acta Anaesthesiol Taiwan. 2012;50:54-8.

14. Kandasami M, Kinninmonth AW, Sarungi M, Baines J, Scott NB. Femoral nerve block for total knee replacement: a word of caution. Knee. 2009;16:98-100.

15. Feibel RJ, Dervin GF, Kim PR, Beaule PE. Major complications associated with femoral nerve catheters for knee arthroplasty: a word of caution. J Arthroplasty. 2009;24(6 Suppl):132-7. 
16. Atkinson HD, Hamid I, Gupte CM, Russell RC, Handy JM. Postoperative fall after the use of the 3-in-1 femoral nerve block for knee surgery: a report of four cases. J Orthop Surg (Hong Kong). 2008;16:381-4.

17. Sorensen JK, Jæger P, Dahl JB, Gottschau B, Stephensen SL, Grevstad U. The isolated effect of adductor canal block on quadriceps femoris muscle strength after total knee arthroplasty: a triple-blinded, randomized, placebo-controlled trial with individual patient analysis. Anesth Analg. 2016;122: 553-8.

18. Grevstad U, Jæger P, Sorensen JK, Gottschau B, Ilfeld B, Ballegaard M, Hagelskjaer M, Dahl JB. The effect of local anesthetic volume within the adductor canal on quadriceps femoris function evaluated by electromyography: a randomized, observer- and subject-blinded, placebo-controlled study in volunteers. Anesth Analg. 2016;123:493-500.

19. Hanson NA, Allen CJ, Hostetter LS, Nagy R, Derby RE, Slee AE, Arslan A, Auyong DB. Continuous ultrasound-guided adductor canal block for total knee arthroplasty: a randomized, double-blind trial. Anesth Analg. 2014;118:1370-7.

20. Davis JJ, Bond TS, Swenson JD. Adductor canal block: more than just the saphenous nerve? Reg Anesth Pain Med. 2009; 34:618-9.

21. Wiesmann T, Piechowiak K, Duderstadt S, Haupt D, Schmitt J, Eschbach D, Feldmann C, Wulf H, Zoremba M, Steinfeldt T. Continuous adductor canal block versus continuous femoral nerve block after total knee arthroplasty for mobilisation capability and pain treatment: a randomised and blinded clinical trial. Arch Orthop Trauma Surg. 2016;136:397-406.

22. Elkassabany NM, Antosh S, Ahmed M, Nelson C, Israelite C, Badiola I, Cai LF, Williams R, Hughes C, Mariano ER, Liu J. The risk of falls after total knee arthroplasty with the use of a femoral nerve block versus an adductor canal block: a double-blinded randomized controlled study. Anesth Analg. 2016;122:1696-703.

23. Rasmussen M, Kim E, Kim TE, Howard SK, Mudumbai S, Giori NJ, Woolson S, Ganaway T, Mariano ER. A retrospective comparative provider workload analysis for femoral nerve and adductor canal catheters following knee arthroplasty. J Anesth. 2015;29:303-7.

24. Patterson ME, Bland KS, Thomas LC, Elliott CE, Soberon JR Jr, Nossaman BD, Osteen K. The adductor canal block provides effective analgesia similar to a femoral nerve block in patients undergoing total knee arthroplasty: a retrospective study. J Clin Anesth. 2015;27:39-44.

25. Memtsoudis SG, Yoo D, Stundner O, Danninger T, Ma Y,
Poultsides L, Kim D, Chisholm M, Jules-Elysee K, Valle AG, Sculco TP. Subsartorial adductor canal vs femoral nerve block for analgesia after total knee replacement. Int Orthop. 2015;39:673-80.

26. Ludwigson JL, Tillmans SD, Galgon RE, Chambers TA, Heiner JP, Schroeder KM. A comparison of single shot adductor canal block versus femoral nerve catheter for total knee arthroplasty. J Arthroplasty. 2015;30(9 Suppl):68-71.

27. Grevstad U, Mathiesen O, Valentiner LS, Jaeger P, Hilsted KL, Dahl JB. Effect of adductor canal block versus femoral nerve block on quadriceps strength, mobilization, and pain after total knee arthroplasty: a randomized, blinded study. Reg Anesth Pain Med. 2015;40:3-10.

28. Zhang W, Hu Y, Tao Y, Liu X, Wang G. Ultrasound-guided continuous adductor canal block for analgesia after total knee replacement. Chin Med J (Engl). 2014;127:4077-81.

29. Shah NA, Jain NP. Is continuous adductor canal block better than continuous femoral nerve block after total knee arthroplasty? Effect on ambulation ability, early functional recovery and pain control: a randomized controlled trial. J Arthroplasty. 2014;29:2224-9.

30. Mudumbai SC, Kim TE, Howard SK, Workman JJ, Giori N, Woolson S, Ganaway T, King R, Mariano ER. Continuous adductor canal blocks are superior to continuous femoral nerve blocks in promoting early ambulation after TKA. Clin Orthop Relat Res. 2014;472:1377-83.

31. Kim DH, Lin Y, Goytizolo EA, Kahn RL, Maalouf DB, Manohar A, Patt ML, Goon AK, Lee YY, Ma Y, Yadeau JT. Adductor canal block versus femoral nerve block for total knee arthroplasty: a prospective, randomized, controlled trial. Anesthesiology. 2014;120:540-50.

32. Jaeger P, Zaric D, Fomsgaard JS, Hilsted KL, Bjerregaard J, Gyrn J, Mathiesen O, Larsen TK, Dahl JB. Adductor canal block versus femoral nerve block for analgesia after total knee arthroplasty: a randomized, double-blind study. Reg Anesth Pain Med. 2013;38:526-32.

33. Burckett-St Laurant D, Peng P, Giron Arango L, Niazi AU, Chan VW, Agur A, Perlas A. The nerves of the adductor canal and the innervation of the knee: an anatomic study. Reg Anesth Pain Med. 2016;41:321-7.

34. Jenstrup MT, Jæger P, Lund J, Fomsgaard JS, Bache S, Mathiesen O, Larsen TK, Dahl JB. Effects of adductor-canalblockade on pain and ambulation after total knee arthroplasty: a randomized study. Acta Anaesthesiol Scand. 2012;56: 357-64.

35. Lund J, Jenstrup MT, Jaeger P, Sorensen AM, Dahl JB. Con- 
tinuous adductor-canal-blockade for adjuvant post-operative analgesia after major knee surgery: preliminary results. Acta Anaesthesiol Scand. 2011;55:14-9.

36. Bendtsen TF, Moriggl B, Chan V, Pedersen EM, Borglum J. Defining adductor canal block. Reg Anesth Pain Med. 2014; 39:253-4.

37. Andersen HL, Zaric D. Adductor canal block or midthigh saphenous nerve block: same same but different name! Reg Anesth Pain Med. 2014;39:256-7.

38. Jaeger P, Koscielniak-Nielsen ZJ, Schroder HM, Mathiesen O, Henningsen MH, Lund J, Jenstrup MT, Dahl JB. Adductor canal block for postoperative pain treatment after revision knee arthroplasty: a blinded, randomized, placebocontrolled study. PLoS One. 2014;9:e111951.

39. Dong CC, Dong SL, He FC. Comparison of adductor canal block and femoral nerve block for postoperative pain in total knee arthroplasty: a systematic review and meta-analysis. Medicine (Baltimore). 2016;95:e2983.

40. Hussain N, Ferreri TG, Prusick PJ, Banfield L, Long B, Prusick VR, Bhandari M. Adductor canal block versus femoral canal block for total knee arthroplasty: a meta-analysis: what does the evidence suggest? Reg Anesth Pain Med. 2016;41: 314-20.

41. Kuang MJ, Xu LY, Ma JX, Wang Y, Zhao J, Lu B, Ma XL. Adductor canal block versus continuous femoral nerve block in primary total knee arthroplasty: a meta-analysis. Int J Surg. 2016;31:17-24.

42. Li D, Ma GG. Analgesic efficacy and quadriceps strength of adductor canal block versus femoral nerve block following total knee arthroplasty. Knee Surg Sports Traumatol Arthrosc. 2016;24:2614-9.

43. Li D, Yang Z, Xie X, Zhao J, Kang P. Adductor canal block provides better performance after total knee arthroplasty compared with femoral nerve block: a systematic review and meta-analysis. Int Orthop. 2016;40:925-33.

44. Zhao XQ, Jiang N, Yuan FF, Wang L, Yu B. The comparison of adductor canal block with femoral nerve block following total knee arthroplasty: a systematic review with metaanalysis. J Anesth. 2016;30:745-54.

45. Shah NA, Jain NP, Panchal KA. Adductor canal blockade following total knee arthroplasty-continuous or single shot technique? Role in postoperative analgesia, ambulation ability and early functional recovery: a randomized controlled trial. J Arthroplasty. 2015;30:1476-81.

46. Akkaya T, Ersan O, Ozkan D, Sahiner Y, Akin M, Gumus H, Ateş Y. Saphenous nerve block is an effective regional technique for post-menisectomy pain. Knee Surg Sports Traumatol Arthrosc. 2008;16:855-8.

47. Hanson NA, Derby RE, Auyong DB, Salinas FV, Delucca C, Nagy R, Yu Z, Slee AE. Ultrasound-guided adductor canal block for arthroscopic medial meniscectomy: a randomized, double-blind trial. Can J Anaesth. 2013;60:874-80.

48. Hsu LP, Oh S, Nuber GW, Doty R Jr, Kendall MC, Gryzlo S, Nader A. Nerve block of the infrapatellar branch of the saphenous nerve in knee arthroscopy: a prospective, doubleblinded, randomized, placebo-controlled trial. J Bone Joint Surg Am. 2013;95:1465-72.

49. Jaeger P, Nielsen ZJ, Henningsen MH, Hilsted KL, Mathiesen O, Dahl JB. Adductor canal block versus femoral nerve block and quadriceps strength: a randomized, double-blind, placebo-controlled, crossover study in healthy volunteers. Anesthesiology. 2013;118:409-15.

50. Kwofie MK, Shastri UD, Gadsden JC, Sinha SK, Abrams JH, $\mathrm{Xu}$ D, Salviz EA. The effects of ultrasound-guided adductor canal block versus femoral nerve block on quadriceps strength and fall risk: a blinded, randomized trial of volunteers. Reg Anesth Pain Med. 2013;38:321-5. 